Explorations of the Syntax-Semantics Interface

Jens Fleischhauer, Anja Latrouite \& Rainer Osswald (eds.)

$\mathbf{d}|\mathbf{u}| \mathbf{p}$ 
Hana Filip, Peter Indefrey, Laura Kallmeyer,
Sebastian Löbner, Gerhard Schurz \& Robert D. Van Valin, Jr. (eds.)

Studies in Language and Cognition 
Jens Fleischhauer, Anja Latrouite \& Rainer Osswald (eds.)

2016

\section{Explorations of the Syntax-Semantics Interface}

$\mathbf{d}|\mathbf{u}| \mathbf{p}$ 


\section{Bibliografische Information}

\section{der Deutschen Nationalbibliothek}

Die Deutsche Nationalbibliothek verzeichnet diese

Publikation in der Deutschen Nationalbibliografie;

detaillierte bibliografische Daten sind im Internet

über http://dnb.dnb.de abrufbar.

\section{(cc) BY-NC-ND}

This work is licensed under the Creative Commons Attribution-NonCommercial-NoDerivs 4.0 License. For details go to http://creativecommons.org/licenses/by-nc-nd/4.0/.

(c) düsseldorf university press, Düsseldorf 2016

http://www.dupress.de

Einbandgestaltung: Doris Gerland, Christian Horn, Albert Ortmann

Satz: Friedhelm Sowa, ETEX

Herstellung: docupoint $\mathrm{GmbH}$, Barleben

Gesetzt aus der Linux Libertine und der Linux Biolinum

ISBN 978-3-95758-000-9

eISBN 978-3-11-072029-7 